\title{
The Value of Support Group Participation in Influencing Adherence to Antiretroviral Treatment among People Living with Human Immunodeficiency Virus (HIV)
}

\author{
Simpson Tumwikirize ${ }^{1 *}$, Kwasi Torpey², Oluwasanmi Adedokun², Tilitope Badru² \\ ${ }^{1}$ Program for Appropriate Technologies in Health, Kampala, Uganda \\ ${ }^{2}$ Family Health International, Abuja, Nigeria \\ Email: ${ }^{*}$ simpson tumwikirize@yahoo.co.uk
}

Received 11 July 2015; accepted 4 September 2015; published 7 September 2015

Copyright (C) 2015 by authors and Scientific Research Publishing Inc.

This work is licensed under the Creative Commons Attribution International License (CC BY). http://creativecommons.org/licenses/by/4.0/

(c) (i) Open Access

\section{Abstract}

Advances in antiretroviral therapy for both Human Immunodeficiency Virus (HIV) treatment and prevention have increased interest in adherence to HIV treatment. This is because the individual patient and public health benefits of antiretroviral treatment (ART) greatly depend on the extent to which people living with HIV (PLHIV) adhere to the prescribed daily dosing regimens. Studies have shown that nonadherence to ART increases drug resistance, morbidity and person-to-person HIV transmission. Public health experts are trying different innovations to enhance ART adherence, including promoting support groups of PLHIV. The purpose of this study was to determine the value of support group participation in enhancing ART adherence. The study used a cross-sectional design to compare ART adherence among PLHIV who participate in support group activities and those who do not. Respondents were adults who were initiated on ART between January 1, 2010 and December 31, 2012. Multistage probability sampling was used to select study sites and respondents. Data was collected using a self-administered questionnaire from 1676 respondents between February and May 2014. Data was analyzed using STATA. Univariate analysis was carried out to generate descriptive statistics, while Chi-square tests were used to examine if there was an association between participation in support group activities and antiretroviral treatment adherence. ART adherence was self-reported by 745/788 (95\%) and 814/888 (92\%) respondents who had ever and had never participated in support group activities respectively. Among respondents who reported nonadherence to ART, 74/117 (8\%) had never participated in support group activities compared to just $43 / 117(5 \%)$ who participated in support group activities. These findings suggest that PLHIV who participate in support group activities are more likely to adhere to ART

\footnotetext{
${ }^{*}$ Corresponding author.
} 
than those who do not participate. This implies that there is value in participating in support groups by PLHIV in terms of ART adherence.

\title{
Keywords
}

\author{
Support Groups, Human Immunodeficiency Virus, Participation, Antiretroviral Therapy, \\ Adherence
}

\section{Introduction}

Success in antiretroviral treatment (ART) for both Human Immunodeficiency Virus (HIV) treatment and prevention has rekindled interest in interventions that enhance ART adherence. This is because the public health impact of ART in reducing HIV transmission and enhancing treatment outcomes greatly depend on the extent to which people living with HIV (PLHIV) adhere to the prescribed daily dosing regimens of antiretroviral medicines. There is concern that a significant proportion of PLHIV on ART do not adhere to their ART [1]

According to the World Health Organization [2], adherence to treatment is the extent to which a patient's history of therapeutic drug-taking coincides with the prescribed treatment. The point that separates adherence from nonadherence is defined as that in the natural history of the disease which makes the desired therapeutic outcome likely (adherence) or unlikely (nonadherence) to be achieved. In ART, adherence of $\geq 95 \%$ is required to achieve durable suppression of viral load. ART adherence may be measured in various ways, for example, selfreports, pharmacy refill logs, pill counting, or a combination of these measures. Researchers have identified multiple factors associated with nonadherence to ART. These fall into broad categories including psychosocial issues, economic factors, substance abuse, co-morbidities including psychiatric disease, low health literacy, and medication-related issues. Many of these factors are modifiable with adherence enhancing interventions [3].

Despite various interventions in ART adherence support, recent studies of PLHIV have reported low adherence rates. In a recent cross-sectional study on HIV infected adults attending an ART clinic in Nigeria, results showed an adherence rate of $85 \%$, while a similar study in Ghana put adherence at 38\% [4] [5]. A near perfect adherence level of $\geq 95 \%$ is required for the effective suppression of HIV/AIDS virus [6]. Excellent adherence is the cornerstone of successful ART because nonadherence leads to drug resistance, treatment failure and death. Also, nonadherence to ART leads to an increase in viral load and increases the risk of HIV transmission from an infected to an uninfected sexual partner. Nonadherence to ART has been associated with risky sexual behaviors. In a study in Atlanta, USA, results show that nonadherent Men who have Sex with Men (MSM) were more likely to have a greater number of sex partners and engage in unprotected sex than those who were adherent [7]. Also, there is evidence that ART reinvigorates sexual desires and sexual activity of PLHIV; and that few PLHIV practice safe sex [8]-[10].

Available literature suggests that HIV-related stigma and nondisclosure of a positive HIV status are major obstacles to ART adherence and reduction in HIV transmission [1]. Nondisclosure of one's positive HIV status has been associated with internalized stigma-negative feelings towards oneself because of HIV positive status. Stigma makes some PLHIV remain silent, alienate themselves, and opt not to enroll in health care services, or hide their antiretroviral drugs (ARVs) from others even when they are faced with life threatening health conditions [11]. In a recent study in rural Nigeria, researchers found a strong association between levels of stigma and adherence to ART. Respondents with a low level of stigma were more likely to adhere to their treatment than those with high levels of stigma [12].

In order to optimize ART adherence, researchers and the public health community continue to explore interventions that reduce stigma and enhance disclosure. Social support interventions are one such strategy. Some studies have established a link between perceived social support and physical and mental health [13]-[16]. Also, social support has been found to promote psychological adjustment in people with high stress due to chronic health problems like HIV/AIDS [17]. In Nigeria, PLHIV support groups are the most common and popular way of providing social support for PLHIV. According to Fanelli \& Moyo [18], a PLHIV support group is an association of PLHIV who come together to share challenges and experiences of living with HIV. It is believed that PLHIV who participate in PLHIV support group activities accrue individual and group benefits. Individual benefits include general supportive counselling, ART adherence counselling, and peer support for addressing other 
HIV-related needs. Group benefits include education/information on HIV/AIDS management, group psychotherapy and experience sharing.

While a number of studies report protective benefits of perceived social support for PLHIV, for example, reduced HIV-related stigma; increased HIV disclosure; safer sexual behaviors; and ART adherence, there is little information about the value of social support group participation in enhancing ART adherence among PLHIV. There remained a gap in the knowledge; whether PLHIV who participate in support group activities are different from those who do not, in terms of adherence to ART. The purpose of this study was to determine the value of participation in PLHIV support groups in ART adherence.

\section{Methods}

\subsection{Study Design}

A cross sectional study was designed to compare ART adherence rates among PLHIV who participate in PLHIV support groups and those who do not. The researchers preferred a cross-sectional design because time and resource constraints precluded a longitudinal design. Data was collected between February and April, 2014. Study respondents included adult PLHIV who were initiated on ART between January 1, 2010 and December 31, 2012, were still in care at the sampled sites, and available to participate in this study. These included both PLHIV who participate in PLHIV support group activities and those who do not. A multistage probability sampling method was used to select study participants.

\subsection{Study Setting}

The study was carried out in Nigeria, in ART sites supported by the Strengthening Integrated Delivery of HIV/ AIDS Services (SIDHAS) project implemented by Family Health International (FHI 360). The SIDHAS project aims to enhance accessibility, quality, integration and government ownership of comprehensive HIV/AIDS services in 15 states in Nigeria. The supported ART sites provide comprehensive HIV/AIDS services to the population from their respective catchment communities. The services include HIV testing and counselling (HTC), prevention of mother-to-child transmission of HIV (PMTCT), ART, and care and support for PLHIV. To enhance retention in care and ART adherence, hospital nurses, doctors and pharmacists provide adherence counselling to all HIV positive patients before initiating them on ART. Adherence counsellors continue to sensitize both pre-ART and ART patients about the benefits of PLHIV support groups and encourage them to become members so as to benefit from continuous adherence support, positive prevention and care services. PLHIV support group members meet at least once a month and carryout activities related to positive prevention, stigma reduction and group psychosocial therapy. Not all PLHIV enrolled in care participate in PLHIV support group activities.

\subsection{Data Collection}

A self-administered questionnaire was developed, pre-tested and used to collect data from the respondents. Most questions were adopted from instruments validated and used in similar settings. A few additional questions were developed to enlist responses peculiar to this study. The questionnaire included questions on: respondents' socio-demographic data (adopted from ORC Macro); PLHIV support group participation (developed by the researchers); and adherence to ART (adopted from a previously validated tool [19].

Respondents were selected randomly from the sampling frame as they came for their ART refill appointments. The research assistants provided the ID numbers of all expected and eligible respondents to the pharmacists. The pharmacists identified clients on the sample list for each day by comparing the numbers on the patient folders or pharmacy order forms and requested them to meet with the research assistants. Research assistants met the referred clients and sought their consent to participate in the study by going through the informed consent form with each eligible respondent. After obtaining informed consent, research assistants provided questionnaires to consenting respondents; provided instructions for filling the questionnaire; and received completed questionnaires on the same day.

\subsection{Measures}

For purposes of this study, a PLHIV support group referred to a formal association of adult PLHIV who met 
monthly either at a health facility or designated place in their community to discuss issues and carryout activities related to individual, group and community management of HIV/AIDS. A PLHIV support group could be health facility or community-based. Membership was defined as being formally registered with a particular PLHIV support group. Participation in PLHIV support group activities was measured as: 1) Having ever attended a PLHIV support group meeting; 2) attending monthly support group meetings. Participation in PLHIV support group activities was thus broadly defined as both being a member and having ever participated in PLHIV support group activities. This measure was a binary variable with response options of Yes or No. Furthermore, respondents were asked to indicate their frequency of participating in monthly PLHIV support group activities over the past 12 months. Responses were grouped in 1 - 5 (low participation) and 6 - 12 (high participation).

The key outcome variable was ART adherence. ART adherence was measured using a three-day drug recall. The following questions were asked to measure the frequency of antiretroviral doses missed in the past three days before the study: 1) "Did you miss your antiretroviral dose yesterday?"; 2) "Did you miss your antiretroviral dose the day before yesterday?”; 3) "Did you miss your antiretroviral dose three days ago?”. These questions were adopted from a tool developed and validated by Gagne \& Naccache [19]. Respondents were asked to indicate by Yes or No whether on any of the past three days they had missed their morning/lunch or evening/dinner doses (for those not yet on fixed dose combinations) or their daily dose (for those on fixed dose combinations). After coding, these values were summed up to get the total number of doses missed in the past three days, divided by the expected number of doses and multiplied by 100 to get the nonadherence score i.e. percentage of doses missed in the last three days. With the nonadherence score, adherence was calculated and defined as $<5 \%$ of doses missed. Therefore, we created a dichotomous variable for adherence: "YES" for taking $\geq 95 \%$ of prescribed antiretroviral drugs versus "NO" for taking $<95 \%$ of prescribed antiretroviral drugs in the last three days before the study.

\subsection{Data Analysis}

All statistical analyses were performed using STATA software (Statacorp. 2011). Descriptive statistics such as median (Interquartile Range; IQR) were used to summarize continuous variables while categorical variables were summarized as proportions. Chi-square test was used to test for associations between categorical variables. P-value $<0.05$ was considered statistically significant.

\subsection{Ethical Approval}

Before data collection, ethical clearance and approval were provided by the Institutional Review Board (IRB) of the University of Nigeria Nsukka, which is accredited by the Nigeria Federal Ministry of Health's Research Ethics Council and the FHI360 Protection of Human Subjects Committee in North Carolina, USA.

\section{Results}

\subsection{Socio-Demographic Characteristics of the Respondents}

A total of 1676 PLHIV participated in this study. The questionnaire response rate was $82 \%$. Of these, $66 \%$ (1111) were females and 32\% (523) were males, while 42 (2\%) did not indicate their sex/gender. At least $75 \%$ (1259) of respondents were below 45 years. The median age of respondents was 37 years (IQR 31 - 44). More than half of the respondents were married (57\%), $44 \%$ completed secondary education, and $68 \%$ were self-employed. The majority (77\%) of the respondents were Christians. Almost two-fifths (636) started ART in 2010 while 29\% (488) and 33\% (552) started in 2011 and 2012 respectively. Table 1 summarizes the socio-demographics of respondents.

\subsection{Membership and Participation in PLHIV Support Groups}

One-third of the respondents indicated that they were members of a PLHIV support group, 8\% had been members but had dropped out, while 6\% had ever participated in PLHIV support group activities but had never been members. Approximately 53\% of respondents had never participated in PLHIV support group activities. As participation in PLHIV support group activities was defined as both being a member and ever participated in PLHIV support group activities, 47\% (788) of respondents were considered to have ever participated in PLHIV 
Table 1. Socio-demographic characteristics of respondents $(\mathrm{N}=1676)^{*}$.

\begin{tabular}{|c|c|c|}
\hline Characteristics & Frequency & Percentage \\
\hline \multicolumn{3}{|l|}{ Gender } \\
\hline Female & 1111 & 66 \\
\hline Male & 532 & 32 \\
\hline None response & 42 & 2 \\
\hline \multicolumn{3}{|l|}{ Age category } \\
\hline $18-30$ & 411 & 25 \\
\hline $31-44$ & 848 & 51 \\
\hline 45 and above & 385 & 23 \\
\hline None response & 32 & 1.9 \\
\hline \multicolumn{3}{|l|}{ Education } \\
\hline Completed primary & 580 & 35 \\
\hline Completed secondary & 737 & 44 \\
\hline Tertiary & 267 & 16 \\
\hline Other & 92 & 5 \\
\hline \multicolumn{3}{|l|}{ Religion } \\
\hline Christianity & 1288 & 77 \\
\hline Islam & 356 & 21 \\
\hline Traditional & 15 & 1 \\
\hline Other & 17 & 1 \\
\hline \multicolumn{3}{|l|}{ Source of income } \\
\hline Farming & 306 & 18 \\
\hline My spouse & 102 & 6 \\
\hline Office work & 272 & 16 \\
\hline Trading/business & 844 & 50 \\
\hline Unemployed & 62 & 4 \\
\hline Non response & 90 & 6 \\
\hline \multicolumn{3}{|l|}{ Marital status } \\
\hline Currently married & 947 & 56 \\
\hline Living with a sexual partner & 29 & 2 \\
\hline Single & 332 & 20 \\
\hline Widowed/separated & 365 & 21 \\
\hline None response & 3 & 0.2 \\
\hline
\end{tabular}

*All percentages presented were calculated based on $\mathrm{N}=1676$.

support group activities against 53\% (888) who had never. This is summarized in Figure 1.

\subsection{Socio-Demographics of Respondents by Support Group Participation Status}

The analysis in Table 2 shows that PLHIV who participated in PLHIV support group activities did not significantly differ from those who did not, in terms of levels of education $(p=0.748)$ and source of income $(p=$ $0.067)$, but significantly differed in marital status $(p \leq 0.001)$, age $(p \leq 0.001)$, sex $(p=0.029)$ and religion $(p=$ 0.034). PLHIV who were not married (single, widowed, separated or divorced) were less likely to participate in PLHIV support group activities than those who were married or living with a sexual partner (37\% vs 67\%). PLHIV support group participation was similar among respondents aged 31 - 44 years (49\%) and those aged 45 and above (50\%). However PLHIV aged below 30 years were less likely to participate in PLHIV support group activities than those aged between 31 and 44 years as well as those over 45 years (21\% vs 54\% vs 25\%). Despite there being more HIV positive women than men, men were more likely to participate in PLHIV support group activities than women (51\% vs 45\%). PLHIV who practiced traditional religion were more likely to participate in PLHIV support group activities than those who practiced Christianity and Islam (80\% vs 47\% vs $46 \%)$.

\subsection{Comparison of ART Adherence Rates by Support Group Participation Status}

Respondents’ responses to questions about adherence to ART were scored to measure their rates of adherence. 


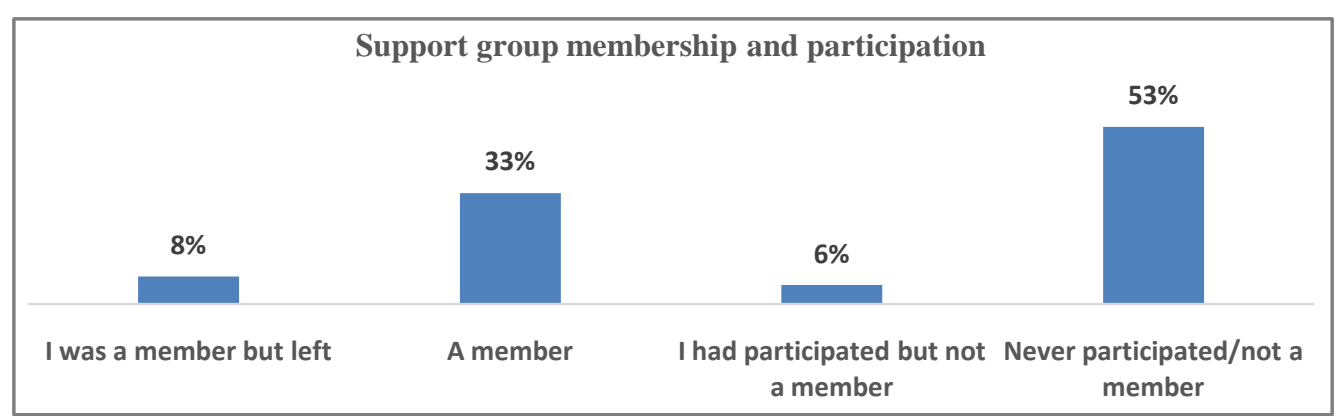

Figure 1. Proportion of PLHIV who belong to and participate in PLHIV support group activities.

Table 2. Socio-demographics by support group participation status.

\begin{tabular}{|c|c|c|c|c|c|}
\hline \multirow{3}{*}{ Variable } & \multicolumn{4}{|c|}{ Support group participation } & \multirow{3}{*}{ p-value } \\
\hline & \multicolumn{2}{|c|}{ NO } & \multicolumn{2}{|c|}{ YES } & \\
\hline & $\mathbf{n}$ & $\%$ & $\mathbf{n}$ & $\%$ & \\
\hline \multicolumn{6}{|l|}{ Gender } \\
\hline Female & 611 & 55 & 500 & 45 & 0.029 \\
\hline Male & 262 & 49 & 270 & 51 & \\
\hline \multicolumn{6}{|l|}{ Age category } \\
\hline $18-30$ & 248 & 60 & 163 & 21 & $<0.001$ \\
\hline $31-44$ & 430 & 51 & 418 & 54 & \\
\hline $45-80$ & 192 & 50 & 193 & 25 & \\
\hline \multicolumn{6}{|l|}{ Education } \\
\hline Completed primary & 305 & 53 & 275 & 47 & 0.748 \\
\hline Completed secondary & 374 & 51 & 363 & 49 & \\
\hline Tertiary & 141 & 53 & 126 & 47 & \\
\hline \multicolumn{6}{|l|}{ Marital status } \\
\hline Currently married & 467 & 49 & 480 & 51 & \\
\hline Living with sex partner & 15 & 52 & 14 & 48 & \\
\hline Single & 195 & 59 & 137 & 41 & $<0.001$ \\
\hline Widowed/separated & 209 & 57 & 156 & 43 & \\
\hline \multicolumn{6}{|l|}{ Religion } \\
\hline Christianity & 685 & 53 & 603 & 47 & \\
\hline Islam & 193 & 54 & 163 & 46 & 0.034 \\
\hline Traditional & 3 & 20 & 12 & 80 & \\
\hline \multicolumn{6}{|l|}{ Source of income } \\
\hline Farming & 154 & 49 & 158 & 51 & \\
\hline My spouse & 60 & 59 & 42 & 41 & 0.067 \\
\hline Office work & 140 & 51 & 132 & 49 & \\
\hline Trading/business & 441 & 52 & 403 & 48 & \\
\hline Unemployed & 42 & 68 & 20 & 32 & \\
\hline
\end{tabular}

Respondents who scored $\geq 95 \%$ were considered adherent while those who scored below 95\% were considered nonadherent. Overall, 1559 (93\%) of the respondents were found adherent and 117 (7\%) not adherent. Generally, there was no significant difference between respondents' socio-demographics and their rates of adherence to ART except for marital status. Respondents' self-reported adherence was highest amongst married respondents (93\%) and lowest amongst respondents living with sexual partner but not married (79\%).

Adherence to ART amongst PLHIV who participated in PLHIV support group activities was reported by 745/788 (95\%) respondents while adherence among PLHIV who never participated in PLHIV support group activities was reported by 814/888 (92\%) respondents. These findings suggest that PLHIV who participate in PLHIV support group activities are more likely to adhere to ART than those who do not participate $(p=0.021)$. The frequency of attending PLHIV support group activities has no association with ART adherence $(p=0.461)$, 
and neither does the year of ART initiation ( $p=0.512$ ). Table 3 summarizes respondents' socio-demographics, PLHIV support group participation status and ART adherence.

\section{Discussion}

Membership of support group offers the opportunity for PLHIV to participate in their care at both the facility and community level. The finding that only 33\% of PLHIV were members of a PLHIV support group is not significantly different from an earlier study in Uganda which put the figure at 30\% [20]. Also, some of the reasons for nonparticipation are similar to findings of a previous study in South Africa. In this study, the reasons given for nonparticipation include lack of time (35\%), lack of information about benefits of PLHIV support groups (29\%), not wanting others to know the respondent's positive HIV status (14\%), lack of transport (6\%), and having other sources of support (5\%). In the South Africa study, the major reasons for nonparticipation in PLHIV support groups were given as: 1) perception that attending PLHIV support group activities would be disclosing their positive HIV status to other people; 2) lack of knowledge of associated benefits; and 3) perceptions that PLHIV support groups lack skills and resources to provide effective psychosocial or other support needed by PLHIV [21]. The apparent low patronage of PLHIV support groups could also be associated with another finding in this study which shows that only $16 \%$ of PLHIV consider PLHIV support group members as their major

Table 3. Association between socio-demographics, PLHIV support group participation and ART adherence.

\begin{tabular}{|c|c|c|c|}
\hline \multirow{3}{*}{ Variable } & \multicolumn{2}{|c|}{ Adherence } & \multirow{3}{*}{ P-value } \\
\hline & YES & NO & \\
\hline & $\mathbf{N}(\%)$ & N (\%) & \\
\hline \multicolumn{4}{|l|}{ Gender } \\
\hline Female & $1038(93)$ & $73(7)$ & 0.325 \\
\hline Male & $490(92)$ & $42(8)$ & \\
\hline \multicolumn{4}{|l|}{ Age Category } \\
\hline$\leq 30$ & $378(92)$ & $33(8)$ & 0.194 \\
\hline $31-44$ & 798 (94) & $50(6)$ & \\
\hline $44-80$ & $353(92)$ & $32(8)$ & \\
\hline \multicolumn{4}{|l|}{ Education } \\
\hline Completed primary & 537 (93) & $43(7)$ & 0.761 \\
\hline Completed secondary & $688(93)$ & $49(7)$ & \\
\hline Tertiary education & $246(92)$ & $21(8)$ & \\
\hline \multicolumn{4}{|l|}{ Marital status } \\
\hline Currently married & $884(93)$ & $63(7)$ & \\
\hline Living with sexual partner & $23(79)$ & $6(21)$ & 0.014 \\
\hline Single & 314 (95) & $18(5)$ & \\
\hline Widowed & $335(92)$ & $30(8)$ & \\
\hline \multicolumn{4}{|l|}{ Source of income } \\
\hline Office work & $255(94)$ & $17(6)$ & \\
\hline Spouse & $92(90)$ & $10(10)$ & 0.255 \\
\hline Farming & $291(93)$ & $21(7)$ & \\
\hline Trading & 790 (94) & $54(6)$ & \\
\hline Unemployed & $54(87)$ & $8(13)$ & \\
\hline \multicolumn{4}{|l|}{ Support group participation status } \\
\hline \multirow{2}{*}{$\begin{array}{l}\text { Participation } \\
\text { Non-participation }\end{array}$} & $745(95)$ & $43(5)$ & 0.021 \\
\hline & $814(92)$ & $74(8)$ & \\
\hline \multicolumn{4}{|l|}{ Frequency of participation } \\
\hline $1-5$ times & 227 (95) & $11(5)$ & \\
\hline $6-12$ times & $391(97)$ & $14(3)$ & 0.461 \\
\hline \multicolumn{4}{|l|}{ Year of ART initiation } \\
\hline 2010 & $596(94)$ & $40(6)$ & \\
\hline 2011 & 455 (93) & $33(7)$ & 0.512 \\
\hline 2012 & $508(92)$ & $44(8)$ & \\
\hline
\end{tabular}


source of social support. This implies that apart from PLHIV support groups, PLHIV have access to other sources of social support and care.

The finding that people aged below 30 years were less likely to participate in PLHIV support group activities than those of higher age is consistent with young people's health seeking behavior [22]. Generally, in Africa, social norms stigmatize young people and inhibit their health seeking behavior. For example, society does not expect unmarried youths to be infected with sexually transmitted infections such as HIV or to be pregnant because they are generally expected to abstain from sex until marriage. Because of this, young people tend to shy away from places where sexual reproductive health services are provided. This may explain why young PLHIV shun PLHIV support groups which are more patronized by older people. Some programs have responded to this challenge by establishing youth friendly service centers.

The finding that men were more likely to participate in PLHIV support group activities than women (51\% vs $45 \%$ ) is a reflection of gender issues in Sub-Saharan Africa. Generally, African men have more "free" time and disposable income than women. Given that $41 \%$ of respondents who do not participate in PLHIV support group activities cited time and money (transport) constraints, it is possible that this is the reason why fewer women than men can afford to participate in PLHIV support group activities.

Study findings indicate that PLHIV who participate in PLHIV support group activities significantly differ from those who do not, in terms of adherence to ART $(p=0.021)$. The finding that PLHIV support group participation is associated with ART adherence is consistent with previous findings which indicate that generally, support from peers, family members or health workers increased ART adherence [23]-[25]. The novel finding from this study is that social support from PLHIV peers, through a PLHIV support group mechanism, has value in terms of ART adherence. This finding is in line with Meaningful Involvement of PLHIV (MIPA)'s principle; that participation of PLHIV in HIV/AIDS prevention, care and treatment enhances their behavior outcomes including reduced stigma, positive HIV status disclosure, reduced sexual risk behavior and ART adherence.

This study presents heterogeneous data from 30 randomly selected health facilities in 10 states of Nigeria spread across five of the six geopolitical zones of the country, thus making the findings generalizable to the whole country. However, the study design had some limitations: Only those respondents who could complete a self-administered questionnaire written in English were included in the study, thus introducing some bias in the selection of respondents. Also, measuring ART adherence was limited to self-reports using a three-day drug recall method; some respondents might have over or under reported their ART adherence-this is inherent in self-behavior. Casual relationships could not be inferred because the study used a cross-sectional design. Further studies addressing these limitations are recommended.

\section{Conclusion}

The study suggests that there is an association between PLHV participation in PLHIV support group activities and adherence to ART; PLHIV who participate in PLHIV support group activities are more likely to adhere to ART than those who do not participate. While the study's design and methodology did not delve into establishing a casual-relationship between PLHIV support group participation and ART adherence, this finding justifies investments in strengthening PLHIV support groups as one of the mechanisms for promoting social support and enhancing ART adherence among PLHIV. HIV/AIDS programs should invest more in strengthening PLHIV support groups as well as enhancing the social support competencies of PLHIV support group members as part of the strategies for enhancing ART adherence.

\section{Acknowledgements}

SIDHAS State Program Managers, SIDHAS Monitoring and Evaluation Technical Officers, All research assistants, Chief Medical Directors of the sampled hospitals, Lisa Duli, Family Health International, Kenya.

\section{References}

[1] United Nations Program on HIV/AIDS (UNAIDS) (2013) Report of the Global AIDS Epidemic 2012. http://www.unaids.org/en/media/unaids/contentassets/documents/epidemiology/2012/gr2012/20121120_UNAIDS_Glo bal_Report_2012_with_annexes_en.pdf

[2] World Health Organization (WHO) (2013) Adherence to Long-Term Therapies-Evidence for Action. http://apps.who.int/medicinedocs/en/d/Js4883e/8.9.1.html 
[3] Enriquez, M. and McKinsey, D.S. (2011) Strategies to Improve HIV Treatment Adherence in Developed Countries: Clinical Management at the Individual Level. Dove Press Journal. HIV/AIDS—Research and Palliative Care, 3, 45-51. http://dx.doi.org/10.2147/HIV.S8993

[4] Obirikorang, C., Selleh, K.P., Abledu, J.K. and Fofie, C.O. (2013) Predictors of Adherence to Antiretroviral Therapy among HIV/AIDS Patients in the Upper West Region of Ghana. ISRN AIDS, 2013, Article ID: 873939. http://dx.doi.org/10.1155/2013/873939

[5] Okoronkwo, I., Okeke, U., Chinweuba, A. and Iheanacho, P. (2013) Nonadherence Factors and Sociodemographic Characteristics of HIV-Infected Adults Receiving Antiretroviral Therapy in NnamdiAzikiwe University Teaching Hospital, Nnewi, Nigeria. ISRN AIDS, 2013, Article ID: 843794. http://dx.doi.org/10.1155/2013/843794

[6] Paterson, D.L., Swindells, S., Mohr, J., Brester, M., Veris, E.N., Squier, C., et al. (2000) Adherence to Protease Inhibitor Therapy and Outcomes in Patients with HIV. Annals of Internal Medicine, 133, 21-30. http://dx.doi.org/10.7326/0003-4819-133-1-200007040-00004

[7] Kalichman, S.C., Ntseane, D., Nthomang, K., Segwabe, M., Phorano, O. and Simbayi, L.C. (2007) Recent Multiple Sexual Partners and HIV Transmission Risks among People Living with HIV/AIDS in Botswana. Sexually Transmitted Infections, 83, 371-375. http://dx.doi.org/10.1136/sti.2006.023630

[8] Dessie, Y. and Deresa, M. (2012) Sexual Practices of HIV-Positive Individuals Attending Antiretroviral Treatment (ART) in Addis Ababa Public Hospitals: Findings from In-Depth Interview. The Pan African Medical Journal, $13,80$.

[9] Kidder, D.P., Bachanas, P., Medley, A., Pals, S. and Nuwagaba-Biribonwoha, H. (2013) HIV Prevention in Care and Treatment Settings: Baseline Risk Behaviours among HIV Patients in Kenya, Namibia, and Tanzania. PLoS ONE, 8, e57215. http://dx.doi.org/10.1371/journal.pone.0057215

[10] Ndziessi, G., Cohen, J., Kouanfack, C., Boyer, S., Moatti, J., Marcellin, F., et al., ANRS 12110/ESTHER Study Group (2013) Changes in Sexual Activity and Risk Behaviors among PLWHA Initiating ART in Rural District Hospitals in Cameroon-Data from the STRATALL ANRS 12110/ESTHER Trial. AIDS Care: Psychological and Socio-Medical Aspects of AIDS/HIV, 25, 347-355.

[11] Overstreet, N.M., Earnshaw, V.A., Kalichman, S.C. and Quinn, D.M. (2013) Internalized Stigma and HIV Status Disclosure among HIV-Positive Black Men Who Have Sex with Men. AIDS Care: Psychological and Socio-Medical Aspects of AIDS/HIV, 25, 466-471. http://dx.doi.org/10.1080/09540121.2012.720362

[12] Omosanya, O.E., Elegbede, O.T., Agboola, S.A., Isinkaye, A.O. and Omopariola, O.A. (2013) Effects of Stigmatization/Discrimination on Antiretroviral Therapy Adherence among HIV-Infected Patients in a Rural Tertiary Medical Centre in Nigeria. Journal of the International Association of Providers of AIDS Care, 13, 260-263.

[13] Bolton, P., Bass, J., Neugebauer, R., Verdeli, H., Loughery, K.F., Wickramaratne, P., et al. (2003) Group Interpersonal Psychotherapy for Depression in Rural Uganda. A Randomized Controlled Trial. Journal of American Medical Association, 289, 3117-3124. http://dx.doi.org/10.1001/jama.289.23.3117

[14] Uchino, B. (2004) Social Support and Physical Health: Understanding the Health Consequences of Relationships. Yale University Press, New Haven, 16-17. http://dx.doi.org/10.12987/yale/9780300102185.001.0001

[15] Bekele, T., Rourke, S.B., Tucker, R., Greene, S., Sobota, M., Koornstra, J., et al. (2013) The Positive Spaces Healthy Places Team. Direct and Indirect Effects of Perceived Social Support on Health-Related Quality of Life in Persons Living with HIV/AIDS. AIDS Care: Psychological and Socio-Medical Aspects of AIDS/HIV, 25, 337-346.

[16] Qiao, S., Li, X. and Stanton, B. (2013) Social Support and HIV-Related Risk Behaviours: A Systematic Review of the Global Literature. AIDS Behaviour, 18, 419-441.

[17] Turner-Cobb, J.M., Gore-Felton, C., Marouf, F., Koopman Kim, P., Israelski, D. and Spiegel, D. (2002) Coping, Social Support, and Attachment Style as Psychosocial Correlates of Adjustment in Men and Women with HIV/AIDS. Journal of Behavioral Medicine, 25, 337-353. http://dx.doi.org/10.1023/A:1015814431481

[18] Fanell, C.W. and Moyo, R.A. (2008) Guidelines for Establishing and Operating Successful Support Groups for People Living with HIV. Catholic Relief Services, Harare.

[19] Gagne, G.C. and Naccache, H. (2003) Validation of a Self-Reported Questionnaire Assessing Adherence to Antiretroviral Medication. AIDS Patient Care STDs, 17, 325-332. http://dx.doi.org/10.1089/108729103322231268

[20] Walakira, E., Kaawa-Mafigiri, D., Byamugisha, J. and Parker Rogers, S. (2012) HIV Perspectives of PLHIV on HIV Prevention: Opportunities and Challenges for Strengthening the Response in Uganda. Family Health International, Washington DC.

[21] Madiba, S. and Canti-Sigaqa, V. (2012) Barriers to Participate in Support Groups for People Living with HIV: A Qualitative Study with Men Receiving Antiretroviral Treatment in an HIV Clinic in Mthatha, South Africa. Global Journal of Health Science, 4,119-128.

[22] Babatunde, A., Tawab, N., Geibel, S., Kalibala, S., Okal, J., Mane, B., et al. (2014) HIV/AIDS Vulnerabilities, Discrimination, and Service Accessibility among Africa’s Youth: Insights from a Multi-Country Study. Population Coun- 
cil, Abuja.

[23] Kamau, T.M., Olsen, V.G., Zipp, G.P. and Clark, M. (2012) The Effectiveness of Social Resource Intervention to Promote Adherence to HIV Medication in a Multidisciplinary Care Setting in Kenya. International Journal of STD \& AIDS, 23, 843-848. http://dx.doi.org/10.1258/ijsa.2012.011472

[24] Sarna, A., Luchters, S., Musenge, E., Okal, J., Chersich, M., Tun, W., et al. (2013) Effectiveness of a Community-Based Positive Prevention Intervention for People Living with HIV Who Are Not Receiving Antiretroviral Treatment: A Prospective Cohort Study. Global Health: Science and Practice, 1, 52-67.

[25] Walstrom, P., Operario, D., Zlotnick, C., Mutimura, E., Benekigeri, C. and Cohen, M.H. (2013) I Think My Future Will Be Better Than My Past: Examining Support Group Influence on the Mental Health of HIV-Infected Rwandan Women. Global Public Health, 8, 90-105. http://dx.doi.org/10.1080/17441692.2012.699539 